\title{
Combination of Gold nanodot on ZnO-NR based Schottky junction platform for SERS Applications
}

\author{
Akhilesh Kumar Gupta1 ${ }^{1}$, Ching-Hsiang Chen ${ }^{4}$, Chao-Sung Lai1, 2, 3 \\ ${ }^{1}$ Department of Electronic Engineering, Chang Gung University, Taoyuan, Taiwan \\ 2 2Department of Nephrology, Chang Gung Memorial Hospital, Taoyuan, Taiwan \\ ${ }^{3}$ Department of Materials Engineering, Ming-Chi University of Technology, New Taipei City, Taiwan \\ ${ }^{4}$ Department of Chemical Engineering, National Taiwan University of Science and Technology, Taiwan \\ Corresponding author's: cslai@mail.cqu.edu.tw
}

\begin{abstract}
:
A well-developed Zinc Oxide Nanorod (ZnO-NR) morphology is synthesized via hydrothermal technique. Enhanced surface morphology of SERS substarte is created by using RTA followed by thermal evaporator resulting in gold nanodot pattern on ZnO-NR, based on schottky junction platform. Which are suitable platform \& morphology for desired enhanced as well as less defect substrate. Synthesized hexagonal ZnO-NR grown with 3um length, 150nm diameter and density 100-NR per $\mu \mathrm{m}^{2}$. Quantified FE-SEM results shows roughness enhancement with gold coating is possible using RTA treatment and Raman analysis shows excellent signal enhancement, which is quite useful for producing practical high sensitive, stable SERS substrate for sensor applications.
\end{abstract}

Key words: ZnO-Nanorod, Nanodot, Schottky junction, Hexagonal morphology, RTA, R6G, SERS Sensor

\section{Introduction}

Surface enhanced Raman scattering (SERS) has attracted considerable attentions due to its high sensitivity, non-destructive nature and better ability to provide information about the structure of the trace materials and fingerprintlike chemical information [1].This technique has shown remarkable attention for detection of low concentration of molecule, with aid of noble metals $(\mathrm{Au}, \mathrm{Ag})$ to build up schottky junction based desired SERS substrate. Among existing semiconductor materials, Zinc Oxide $(\mathrm{ZnO})$ has wide band gap, wurtzite crystal structure, and high optical gain. In this work the role of gold nanodot for SERS application via thermal evaporator followed by synthesis of $\mathrm{ZnO}-\mathrm{NR}$ using hydrothermal process and using of RTA to get better improvement of suitable schottky junction based surface for enhanced signal.

\section{Experimental procedure}

Detailed synthesis process of $\mathrm{ZnO}-\mathrm{NR}$ and explanation of SERS process flow shown in figure (1). ZnO-NR were grown on ITO glass at $95^{\circ} \mathrm{C}$, followed by coating of $5 \mathrm{Mm}$ zinc acetate dehydrate in ethanol solution in form of seed layer. After coating of solution on ITO substrate was annealed at $500^{\circ} \mathrm{C}$ in air for 30 minutes to generate $\mathrm{ZnO}$ seed layer on ITO glass. Stock solutions of zinc acetate dehydrate \&HMTA was prepared and these solution was transferred into Teflon lined sealed stainless steel autoclaves and maintained at temperature of $95^{\circ} \mathrm{C}$ with 8 hours [2]. The further generation of hot-spot on SERS substrate, RTA $\left(650^{\circ} \mathrm{C}\right)$ was used followed by gold (3nm) deposition on ZnO-NR for desirable R6G $(1 \mu \mathrm{M})$ detection.

\section{Results \& discussion}

Figure (1) Overall schematic process involving the fabrication of SERS substrates including schottky model based, wherein initially ZnO-NR are grown on ITO glass and decorated with gold film to create gold nanodot using RTA. Our strategy is to inter particle spacing of the SERSactive material on the substrate surface known to create 'hot-spots' for enhancement. In order to get greater enhancement of signal, Rapid Thermal Anneal (RTA) treatment is applied for the improvement of surface roughness [3]. Moreover energy diagram of schottky energy diagram reveal that the levels of first ionizations of zinc interstitials and oxygen vacancies and electron traps were distributed between Ec of $0.05 \mathrm{eV}$ and $-0.5 \mathrm{eV}$. However, the Fermi level $\left(E_{F}\right)$ in the $\mathrm{ZnO} N R s$ can move to upper levels close to Ec. Since the work function of $\mathrm{ZnO}$ becomes lower than that of $\mathrm{Au}$, the electrons are transferred from the $\mathrm{ZnO} \mathrm{NRs}$ to $\mathrm{Au}$ resulting in the SPR coupling model. Figure (2) 
shows FE-SEM morphology of ZnO-NR (A), Au/ZnO-NR without RTA (B), with RTA (C). EDX confirm elements before and after gold deposition on ZnO-NR. After treatment of RTA the surface is roughened and formed gold nanodot (inset figure $2 \mathrm{C}$ ) on $\mathrm{ZnO}-\mathrm{NR}$. Figure (3A) shows laser points at 10 position on SERS surface, and it exhibits uniformity of roughened surface, Figure (3B) confirms highly sensing property and exhibit detection of lower concentration R6G $(1 \mu \mathrm{M})$.

\section{Conclusions}

This study demonstrates the development of highly sensitive roughened SERS surface based schottky junction platform with less defect, to create gold nanodot followed by RTA, on $\mathrm{ZnO}-\mathrm{NR}$ and its suitability for detection of lower concentration of R6G $(1 \mu \mathrm{M})$. Which are cost effective, highly sensitive and stable for sensor applications.

\section{Acknowledgement}

This research is supported by contract number MOST 105-2632-E-182-001, MOST 105-2221E-12-066 and CMRPD2F0021, CMRPD3D0112

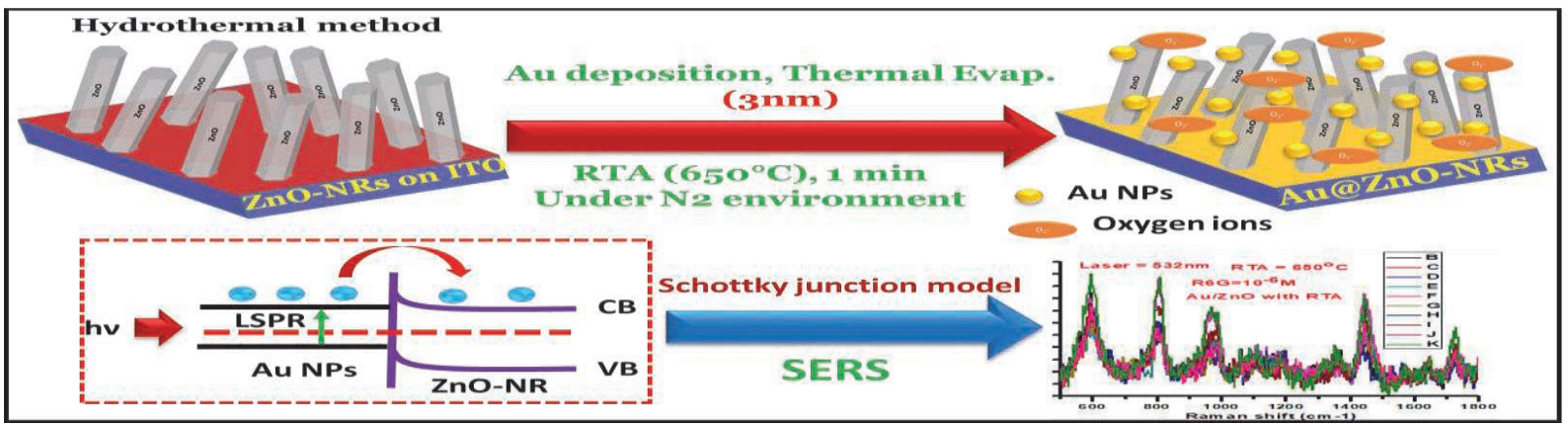

Figure (1) Schematic diagram of overall process and Schottky junction model

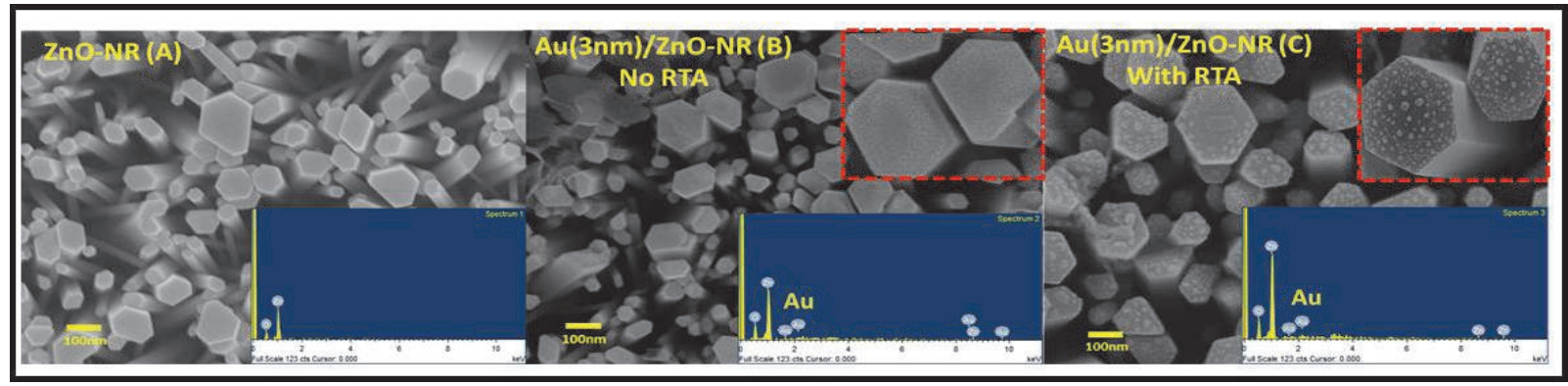

Figure (2) Morphology results (A) ZnO-NR (B) Au@ZnO-NR (C) Gold nucleation over ZnO-NR; EDX shows elemental confirmation with \& without gold (Inset figure shows roughened surface)

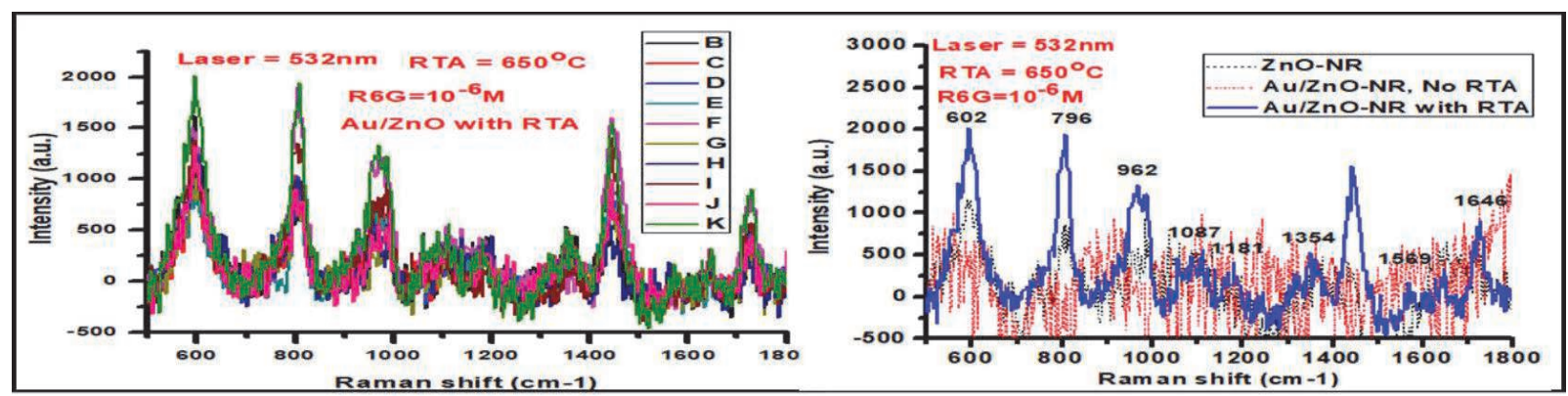

Figure (3) Raman measurements (A) Laser point at 10 position on SERS substrate (B) Signal response of gold nucleation over ZnO-NR with RTA $\left(650^{\circ} \mathrm{C}\right)$ detection of $1 \mu \mathrm{M} R 6 \mathrm{G}$.

\section{References}

[1] W. E. Smith, "Practical understanding and use of surface enhanced Raman scattering/surface enhanced resonance Raman scattering in chemical and biological analysis," Chem. Soc. Rev., 37, pp. 955-964, (2008); doi: 10.1039/B708841H

[2] Liang-Yih Chen, Yu-Tung Yin, Ching-Hsiang Chen, and Jau-Wern Chiou, Influence of Polyethyleneimine and Ammonium on the
Growth of ZnO Nanowires by Hydrothermal Method, J. Phys. Chem. C, 115, 20913-20919,( 2011);doi: 10.1021/jp2056199

[3] A.Purwidyantri, Imène El-Mekki, C.S.Lai, "Tunable Plasmonic SERS "Hotspots" on AuFilm over Nanosphere by Rapid Thermal Annealing," IEEE Transactions on Nanotechnology, Volume: 16, Issue: 4, pp. 551 559, (2017); doi: 10.1109/TNANO.2016.2647263 